\title{
Further Structural Analysis of Cr(III) Oligomers in Weakly Acidic Solutions
}

\author{
Zhicheng Zhang ${ }^{\mathrm{a}, \mathrm{b}, *}$, Sue B. Clark, ${ }^{\mathrm{a}, *}$ Linfeng Rao, ${ }^{\mathrm{b}, *}$ Geoffrey J. Puzon ${ }^{\mathrm{c}}$ and Luying Xun ${ }^{\mathrm{c}}$

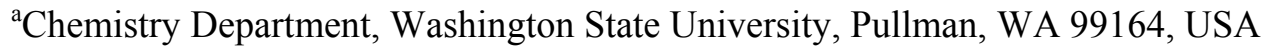 \\ ${ }^{b}$ Chemical Sciences Division, Lawrence Berkeley National Laboratory, Berkeley, CA 94720, USA \\ ${ }^{\mathrm{c}}$ Center for Multiphase Environmental Research, Washington State University, Pullman, WA 99164, USA
}

Author email address: lxzhang@lbl.gov

*Corresponding authors: Sue B. Clark, s_clark@wsu.edu; Linfeng Rao, 1rao@lbl.gov; Zhicheng Zhang, lxzhang@1bl.gov, Telephone: (001) 5104865141, fax: (001) 5104865596 


\begin{abstract}
Three chromium(III) hydrolytic oligomers (monomer, dimer, and trimer) were isolated via cation-exchange from weakly acidic solutions, and their structures were elucidated with multiple techniques including UV-visible and Infrared (IR), electron paramagnetic resonance (EPR), and extended X-ray absorption fine structure (EXAFS) spectroscopy. The results indicate that the monomer has an octahedral symmetry with six water molecules at its six apices. In the dimer, two Cr(III) ions, each of which has an octahedral coordination symmetry distorted by the stretching along the $\mathrm{Cr}-\mathrm{Cr}$ joining axis, are doubly bridged by two hydroxyl groups. Suggested by the data from EXAFS and EPR, the trimer in solution may form with an irregular triangular configuration, in which two $\mathrm{Cr}$ (III) ions are doubly bridged by hydroxyl groups and the third $\mathrm{Cr}(\mathrm{III})$ ion is connected to each of the two through single-hydroxyl bridging.
\end{abstract}

Key words: chromium, oligomer, hydrolysis, EPR, EXAFS 


\section{Introduction}

The motivation behind the present study is the desire to obtain structural information for the small hydrolytic species of $\mathrm{Cr}(\mathrm{III})$ cation, i.e., monomer, dimer and trimer, existing in weakly acidic solutions. These small species are basic motifs leading to the formation of high hydrolytic oligomers of $\mathrm{Cr}(\mathrm{III})[1,2]$. Therefore, their structural information helps analyze the high oligomer structures and understand the mechanism of $\mathrm{Cr}(\mathrm{III})$ hydrolysis and polymerization in aqueous solutions.

The hydrolytic oligomerization of aquo Cr(III) ion has been extensively investigated [110] since Bjerrum postulated the presence of dimeric species from $\mathrm{pH}$ titration experiments in 1908 [11]. Taking advantage of the kinetically inert behavior of chromic species in solutions, a certain number of individual oligomers have been isolated from refluxed, aged, or alkalinized chromic solutions by using ion exchange chromatography. In 1959, monomer and dimer of $\mathrm{Cr}(\mathrm{III})$ were first isolated and identified from the refluxed chromic perchlorate solutions with a Dowex-50 cation exchange resin [4]. By using a different eluting agent, a trimeric species was also obtained later from a similar refluxed chromic perchlorate solution [7]. In the early 1980's, a new cation exchange resin, Sephadex SP C-25, started to be used for such a separation. As a result, additional oligomeric fractions, purported to be the tetramer, pentamer, and hexamer, were successfully isolated from the alkalinized chromic solutions [1]. Those separation efforts not only yielded isolated $\mathrm{Cr}$ (III) oligomers, but also provided convenience for a structural analysis of the oligomers so that the mechanism behind the polymerization could be well approached. It is generally believed that $\mathrm{Cr}(\mathrm{III})$ aquo ions are readily polymerized through hydroxyl (or oxo) bridging, forming a variety of hydrolytic oligomers in aqueous solutions. 
Structures of various $\mathrm{Cr}(\mathrm{III})$ oligomers have been studied for decades [5-7,12]. However, even for small hydrolytic oligomers, their structural details in solutions remain unknown. For example, two distinctively different configurations, linear or triangular, were postulated for the structure of $\mathrm{Cr}(\mathrm{III})$ trimer. In the early 1980's, Finholt et al. conducted the EPR and magnetic susceptibility work. The data analysis seemed to suggest a possibility of the existence of triangular configuration but could not reach a decisive conclusion for it [7]. In the late 1990's, the trimeric crystalline complex was synthesized from solutions through the self-assembly process with a capping ligand (p-sulfonatocalix[4,5]arenes) [12]. While the resolved crystal structure indicated that three chromium atoms of the trimer possess an irregular triangular configuration, a question remains: Does this structure represent the trimer structure in the solution? Beside the existence of large uncertainties in its crystallographic elucidation [12], possible structural changes during the self-assembly crystallization may cast doubt on its representability.

Since the early 2000's, we have studied the oligomerization of $\mathrm{Cr}(\mathrm{III})$ in solutions using techniques including EXAFS [13] and capillary electrophoresis [14]. Although the chromium geometric positions in the dimer and trimer have been obtained by EXAFS [13], their structural details is lacked. In the present study, we systematically evaluated the structures of $\mathrm{Cr}(\mathrm{III})$ monomer, dimer, and trimer by integration of multiple analytical tools including EPR, EXAFS, UV-visible, and IR spectroscopy. The dimer presented a unique EPR spectrum, demonstrating its double hydroxyl bridging structure. A substantial contribution was made to understand a structure of the timer. The combined data of EPR and EXAFS confirmed its irregular triangular configuration. 


\section{Results and Discussion}

2.1 UV-visible and IR spectroscopy

The UV-visible absorption spectra of three isolated Cr(III) oligomers (monomer, dimer and trimer) from aqueous solutions are shown in Figure 1 and their major features listed in Table 1. Two absorption bands are assigned to the $\mathrm{Cr}(\mathrm{III}) \mathrm{d}-\mathrm{d}$ transitions of $\mathrm{A}_{2 \mathrm{~g}}(\mathrm{~F})-\mathrm{T}_{1 \mathrm{~g}}(\mathrm{~F})$ and $\mathrm{A}_{2 \mathrm{~g}}(\mathrm{~F})$ $-\mathrm{T}_{2 \mathrm{~g}}(\mathrm{~F})$, respectively (c.f., Figure 1 ). The measured UV-visible spectra as well as their features agree with those reported in the literature $[1,6-8]$. It is thus evident that the desired oligomers (monomer, dimer and trimer) were successfully obtained through the chromatographic column.

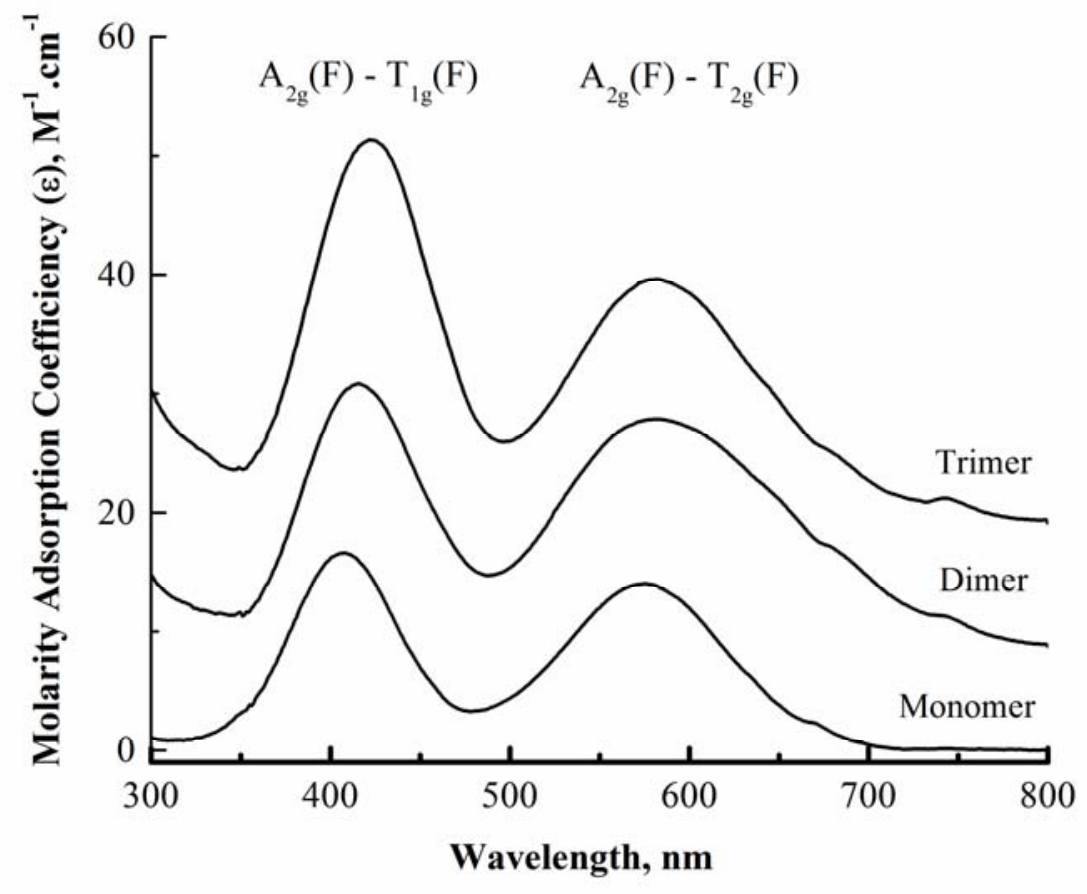

Figure 1 UV-visible absorbance spectra of $\mathrm{Cr}(\mathrm{III})$ oligomer solutions

Two d-d transition bands, as seen in Table 1, shift towards longer wavelength from monomer $\left(\lambda_{1}=575 \mathrm{~nm}, \lambda_{2}=408 \mathrm{~nm}\right)$ to dimer $(580 \mathrm{~nm}, 416 \mathrm{~nm})$ and to trimer $(582 \mathrm{~nm}, 424 \mathrm{~nm})$, and their extinction coefficients also increase along the oligomerization $\left(\varepsilon_{1}=14.0\right.$ (monomer), 
17.8 (dimer) and $19.6 \mathrm{M}^{-1} \mathrm{~cm}^{-1}$ (trimer); $\varepsilon_{2}=16.7$ (monomer), 20.9 (dimer) and $29.4 \mathrm{M}^{-1} \mathrm{~cm}^{-1}$ (trimer)). These variations can be attributed in general to the structural difference of the oligomers and the magnetic coupling presented in dimer and trimer. The $\mathrm{Cr}(\mathrm{III})$ ion in a ligand field tends to maintain its octahedral symmetry, regular or slightly distorted, with six coordinates at individual apices. The distortion, even though slight, may alter the positions and intensities of the transition bands [1,6-8]. The $\mathrm{Cr}(\mathrm{III})$ monomer holds six coordinated water molecules and possesses almost a regular octahedral symmetry with six oxygen atoms on the apices. In the dimer and trimer, however, the hydroxyl bridging may distort the symmetry, thereby shifting the absorption bands and varying the intensities. Also, for these spin-forbidden d-d transitions, the presence of the $\mathrm{Cr}(\mathrm{III})-\mathrm{Cr}(\mathrm{III})$ magnetic exchange coupling in the dimer and trimer, which is discussed in the following section, may relax the selection rule to some extent and thus enhance the transitions, resulting in the increase of absorbance intensity. An effect of the magnetic coupling on visible spectra of the transition metal ions has been well described in the reference [15].

Table 1 Spectroscopic characteristics of Cr(III) oligomers

\begin{tabular}{c|ccccc|c}
\hline \multirow{3}{*}{ Cr(III) Species } & \multicolumn{5}{|c|}{ UV-visible absorbance spectra } & IR-spectra \\
& $\begin{array}{c}\lambda_{1} \\
\mathrm{~nm}\end{array}$ & $\begin{array}{c}\mathcal{M}^{-1} \mathrm{~cm}^{-1} \\
\lambda_{2}\end{array}$ & $\begin{array}{c}\lambda_{2} \\
\mathrm{~nm}_{2}\end{array}$ & $\mathcal{M}_{2} / \mathrm{cm}_{1}$ & $\begin{array}{c}\text { Cr-O rocking band } \\
\mathrm{cm}^{-1}\end{array}$ \\
\hline Monomer & 575 & 14.0 & 408 & 16.7 & 1.19 & 528 \\
Dimer & 580 & 17.8 & 416 & 20.9 & 1.17 & 472 \\
Trimer & 582 & 19.6 & 424 & 29.4 & 1.60 & 481 \\
\hline
\end{tabular}

The IR spectra of $\mathrm{Cr}(\mathrm{III})$ oligomers were taken on solid samples precipitated from the solutions of isolated oligomers by using pyridine and acetone. A frequency of the $\mathrm{Cr}$-O rocking 
band is summarized in Table 1. It is also noted that the IR spectra (data not shown) of all three oligomers found no IR bands of pyridine or acetone, indicating that the samples were free of pyridine or acetone.

An important finding from the IR work is that the $\mathrm{Cr}-\mathrm{O}$ rocking band among the oligomers varies (c.f., Table 1). The rocking frequency of the Cr-O bond decreases appreciably from monomer $\left(528 \mathrm{~cm}^{-1}\right)$ to dimer $\left(472 \mathrm{~cm}^{-1}\right)$ and trimer $\left(481 \mathrm{~cm}^{-1}\right)$. This trend can be attributed to the presence of bridging hydroxyl groups in the dimer and trimer. The oxygen atom of the bridging hydroxyl group binds two $\mathrm{Cr}(\mathrm{III})$ ions simultaneously, resulting in a weaker $\mathrm{Cr}$ Ohydroxyl bond than that of $\mathrm{Cr}-\mathrm{O}_{\text {water. }}$ As a result, the rocking frequency decreases from the monomer to the dimer and trimer.

\subsection{Electron Paramagnetic Resonance (EPR) spectroscopy}

The EPR spectra of three isolated oligomers at two temperatures $(1.4$ and $77 \mathrm{~K})$ are depicted in Figure 2. They contain a great deal of information on their spin properties and structures.

\subsubsection{Monomer EPR}

The EPR spectra of monomer (A1 and A2 in Figure 2) are similar to that reported in the early work [6]. They present a single, identical, nearly symmetric band at both 1.4 and $77 \mathrm{~K}$. It is reasonably assumed that the $\mathrm{Cr}(\mathrm{III})$ ion in monomer possesses an isotropic Zeeman splitting factor $(\boldsymbol{g})$. The $g$ value can be determined to be 1.976 from the peak position. The above observation and assumption agree with the theoretical prediction of $\mathrm{Cr}$ (III) EPR in the monomer. The electronic configuration of $\mathrm{Cr}(\mathrm{III})$ is $\operatorname{Ar} 3 \mathrm{~d}^{3}$ with a spin of $S=3 / 2$. As shown in Scheme Ia, 
the $\mathrm{Cr}(\mathrm{III})$ ion of monomer is located in an octahedral crystal field, leading to a Kramer doublet (Ms $= \pm 1 / 2$ ) lowest in energy due to its large spin-orbit coupling. As a result, the EPR measurement of monomer is expected to yield a single, isotropic band, corresponding to the Zeeman split of the Kramer doublet.

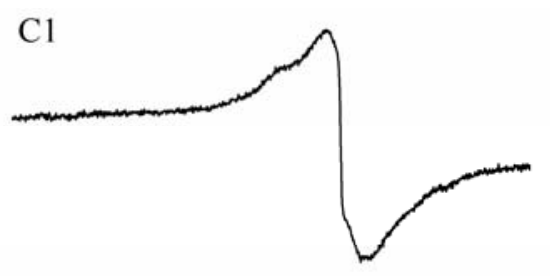

$\mathrm{C} 2$

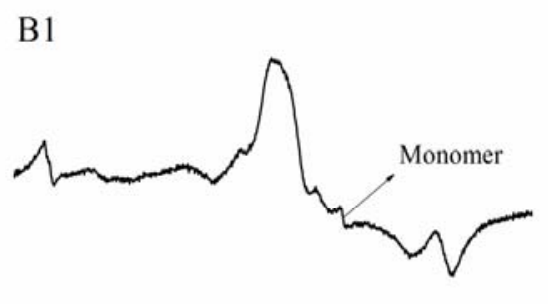

B2
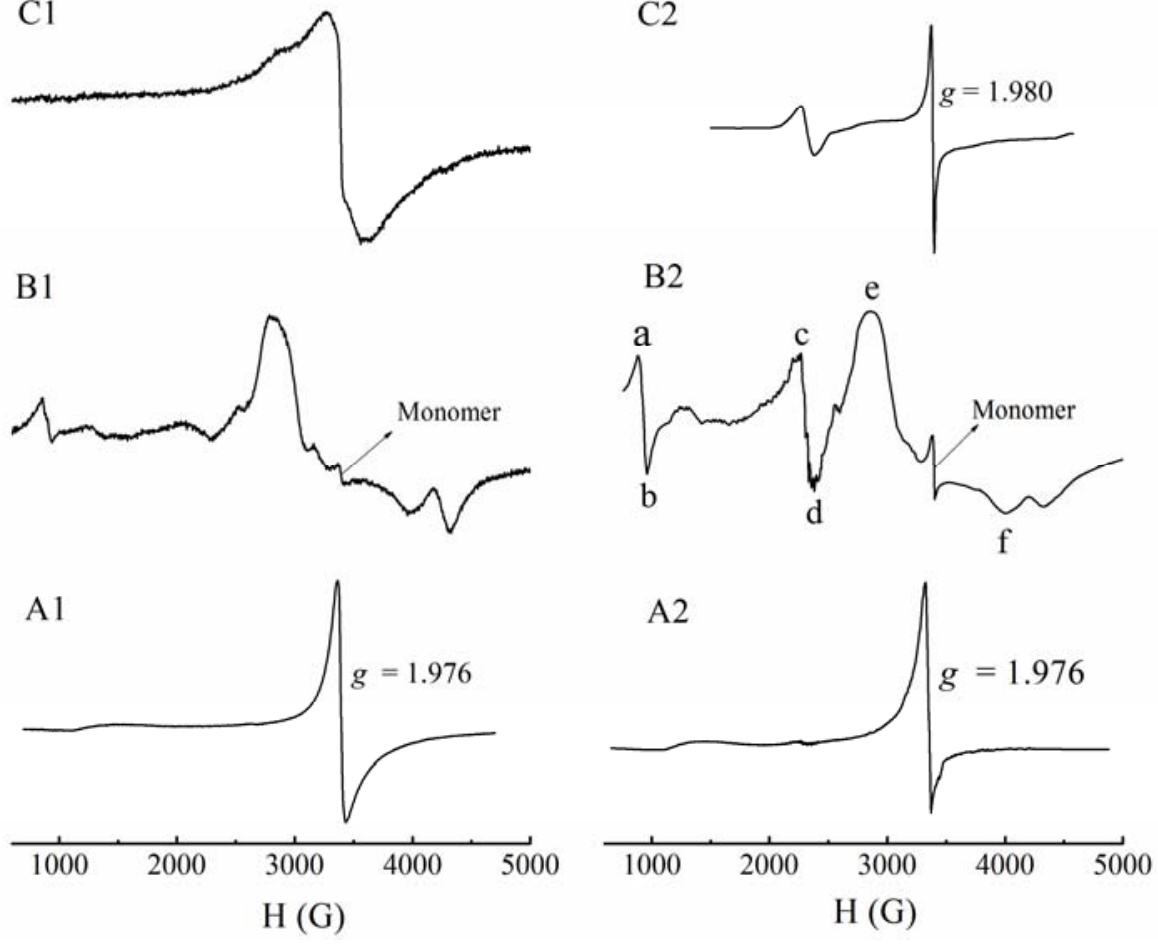

Figure 2 EPR spectra of Cr(III) oligomers. Monomer: A1 (77 K) and A2 (1.4 K); Dimer: B1(77 K) and B2 (1.4 K); Trimer: $\mathrm{C} 1(77 \mathrm{~K})$ and $\mathrm{C} 2(1.4 \mathrm{~K})$.

The $g$ value of $\operatorname{Cr}(\mathrm{III})$ is affected by the spin-orbit coupling constant $(\lambda)$ and the ligand field splitting energy $(\Delta)$. In an octahedral field, the $g$ value of $\mathrm{Cr}(\mathrm{III})$ is given by [16]

$$
g=2.0023-8 \lambda / \Delta
$$

The value of $\lambda$ was reported [17] as $91 \mathrm{~cm}^{-1}$ and the value of $\Delta$ equals to the electronic transition energy between $A_{2 g}(F)$ and $T_{2 g}(F)[16]$. From the absorption spectrum of monomer shown in Figure 1, the $\Delta$ value can be determined to be $17390 \mathrm{~cm}^{-1}$. By using equation (1), the $g$ value is 
thus calculated to be 1.960. The small discrepancy between the measured (1.976) and the calculated (1.960) can be attributed to the binding effects that reduce the effective value of the spin-orbit coupling and the orbital moment [18].

The EPR features of monomer, in combination of its UV-visible spectrum, suggest that the $\mathrm{Cr}$ (III) monomer in solution most likely holds a symmetric octahedral structure as shown in Scheme Ia.

\subsubsection{Dimer EPR}

EPR spectra of the dimer show multiple, broad transition bands (B1 and B2 in Figure 2) and dramatically differ from those of the monomer. Prior to analyzing the dimer EPR, two notes are made here. First, the literature has indicated that dimer holds two different configurations, single (hydroxyl) bridged dimer (SBD) and double (hydroxyl) bridged dimer (DBD) [6]. Only DBD was isolated and studied in this work. Secondly, the measured dimer spectra contain a small feature of the monomer EPR. This implies that the dimer sample might have a small amount of monomer. However, the major features of the dimer EPR are not altered or distorted by this impurity.

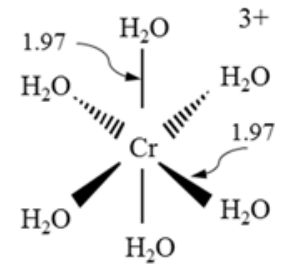

(a)

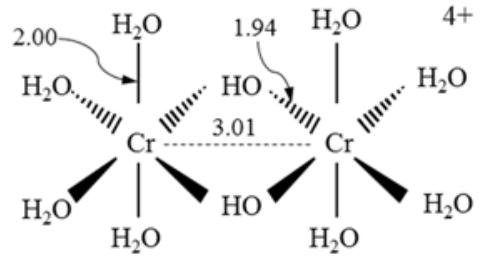

(b)

Scheme I Proposed structures of monomer and dimer 
The unique EPR features of dimer are attributed to the effect of its double hydroxyl bridging. The bridging not only distorts an octahedral coordination symmetry of individual $\mathrm{Cr}(\mathrm{III})$ ions but also enables two $\mathrm{Cr}(\mathrm{III})$ centers to have magnetic exchange.

The exchange coupling of certain transition metals (Fe, Mn and $\mathrm{Cr}$ ) through bridging groups has been investigated [6,19-22]. For such an oligomeric compound, the spin properties are defined by combined spins of the coupled metal atoms (total spin). For a di-Cr(III) coupling system, the spin Hamiltonian is described as follows [6]:

$$
H=g H_{z} S_{z}^{\prime}+2 J S_{1} S_{2}
$$

where $g$ and $H_{z}$ have their usual meaning, $J$ is the exchange coupling constant, $\mathrm{S}_{1}$ and $\mathrm{S}_{2}$ are the spin vectors of two $\mathrm{Cr}(\mathrm{III})$ ions, $S_{\mathrm{z}}{ }^{\prime}$ is the z component of total spin $S^{\prime}$. The dimer with $S^{\prime}=3$ presents four spin states: a ground state $\left(S^{\prime}=0\right)$, three excited states $\left(S^{\prime}=1,2,3\right)$, which are separated by the spin coupling energy [6].

The coupling strength varies with different metals and bridging groups. Thompson et al. studied the exchange coupling between two $\mathrm{Cr}$ (III) ions in the hydroxyl bridged dimer (SBD and DBD) by measuring a magnetic susceptibility [6]. The coupling constants of the SBD and the DBD were found, respectively, to be 11 and $5.2 \mathrm{~cm}^{-1}$. Apparently, the DBD presents very weak coupling and its energy difference between the lowest excited state $\left(S^{\prime}=1\right)$ and the ground state $\left(\mathrm{S}^{\prime}=0\right)$ is only $10.4 \mathrm{~cm}^{-1}(2 J)$. With such a small energy gap, the excited state is accessible even at extremely low temperatures. Therefore, the EPR signals of the dimer, which were observed at $1.4 \mathrm{~K}$ (spectrum B2), most likely represent the spin transitions of its excited state $\left(\mathrm{S}^{\prime}=1\right)$ because its singlet ground state $\left(\mathrm{S}^{\prime}=0\right)$ is EPR-silent.

A carful inspection of spectrum B2 reveals an existence of three major broadened bands, which were denoted by a-b, c-d and e-f in the figure. An accurate interpretation of those bands is 
impossible from the currently available data and also beyond the scope of this work. But, one speculation is given below. As discussed above, suppose these three bands correspond to spin transitions of the spin $S^{\prime}=1$ state. On this spin state, two unpaired electrons undergo a magnetic dipole-dipole interaction, resulting in the zero-field splitting. Furthermore, the symmetry of the dimer molecule is not cubic but uniaxial (c2v) due to the stretching of double hydroxyl groups so that the zero-field splitting is observable. We thus attribute the three bands to two $\Delta \mathrm{Ms}^{\prime}= \pm 1$ transitions and one $\Delta \mathrm{Ms}^{\prime}= \pm 2$ transition. The possible assignment of individual bands follows: Transition a-b: $\mathrm{Ms}^{\prime}-1 \rightarrow 1$; transition c-b: Ms' $0 \rightarrow 1$; transition e-f: $\mathrm{Ms}^{\prime}-1 \rightarrow 0$. Also, the broadening of the bands may be accounted for by the rapid relaxation due to the flexible structure of dimer. The mechanism of such zero-field splitting and spin transitions has been described in the chapter 9 of reference 15 and no further discussion is given here.

In summary, the EPR of dimer becomes complicated because of the presence of $\mathrm{Cr}(\mathrm{III})$ $\mathrm{Cr}(\mathrm{III})$ magnetic coupling.. At the temperature of $1.4 \mathrm{~K}$, it presents three major bands, possibly corresponding to three spin transitions of the lowest excited spin state $\left(S^{\prime}=1\right)$. The EPR result supports the structure of dimer with double hydroxyl bridging as is shown in scheme Ib.

\subsubsection{Trimer EPR}

Spectra of the trimer EPR ( $\mathrm{C} 1$ and $\mathrm{C} 2$ in Figure 2) exhibit interesting spin properties. Its features significantly change with temperature, varying from a single broadened band at $77 \mathrm{~K}$ to two well-separate peaks at $1.4 \mathrm{~K}$.

The trimer could have linear or triangular configurations as shown in Scheme II. However, the EXAFS results from this work, which is discussed in the next section, exclude the linear configuration (Scheme IIa). Therefore, only two triangular configurations (Scheme IIb, c) 
are necessarily discussed with observed EPR features. These two configurations differ in the bridging of the chromium atoms. Scheme IIb represents a structure called a regular triad [20], in which the three $\mathrm{Cr}$ (III) ions are equally connected through single-hydroxyl bridging and a central oxygen atom. In scheme IIc, two $\mathrm{Cr}$ (III) ions are connected through double-hydroxyl bridging and the third $\mathrm{Cr}$ (III) ion is connected to each of the two through single-hydroxyl bridging. For convenience, this structure is referred to as an irregular triad.

For both triad configurations, the spin Hamiltonian can be written as [23-25]

$$
H=2 J_{0}\left(S_{1} S_{2}+S_{1} S_{3}\right)+2\left(J_{0}+J_{1}\right) S_{2} S_{3}
$$

where $S 1, S 2$ and $S 3$ are the spins of three $\mathrm{Cr}(\mathrm{III})$ ions, and $J_{0}$ and $J_{1}$ are the coupling constants. If $J_{1}=0$, the isotropic antiferromagnetic coupling leads to the formation of multiplets of total spins $\left(S^{\prime}\right): 1 / 2,3 / 2,5 / 2,7 / 2,9 / 2$ with relative energies of $0,3 J_{0} / 2,4 J_{0}, 15 J_{0} / 2,12 J_{0}$, respectively [25]. The existence of $J_{1}$ complicates the sequence of spin energy levels and breaks the degeneracy of individual levels [25].

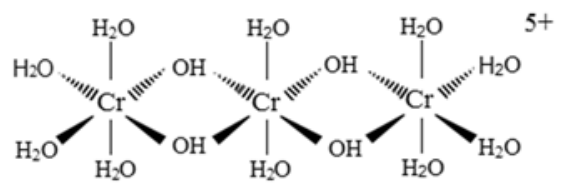

(a)

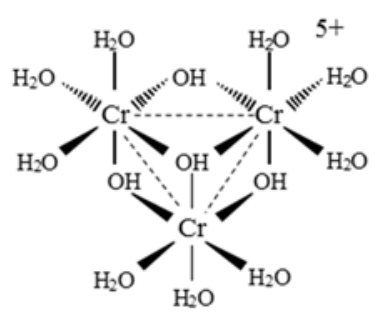

(b)

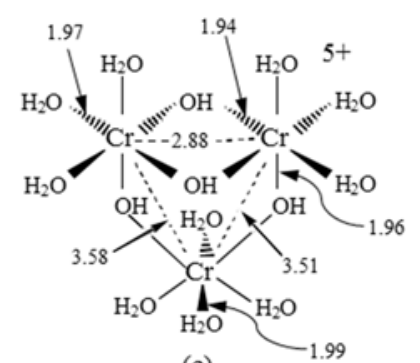

(c)

Scheme II Proposed configurations of trimer

To help determine the configuration of Cr(III) trimer, the observed EPR spectra are used to compare to those of two well-characterized $\mathrm{Cr}(\mathrm{III})$ compounds reported in the literature [20, 
23, 25-29]. The first compound is tri-Cr(III) acetate $\left(\left[\mathrm{Cr}_{3} \mathrm{O}\left(\mathrm{CH}_{3} \mathrm{COO}\right)_{6}\left(\mathrm{H}_{2} \mathrm{O}\right)_{3}\right] \mathrm{Cl}^{-} 6 \mathrm{H}_{2} \mathrm{O}\right)$ $[23,26,27]$. In this molecule, three $\mathrm{Cr}(\mathrm{III})$ ions are mutually bridged by two carboxylate groups and individually bind to the central oxygen atom, forming a regular triad structure with the $\mathrm{C}_{3 \mathrm{v}}$ symmetry. The EPR of this compound has been studied experimentally and theoretically $[20,25,28]$. The measured EPR spectrum varies with temperature. At the temperatures of $300 \mathrm{~K}$ and $77 \mathrm{~K}$, a broadened band with small shoulders was obtained. As the temperature was decreased to $4.2 \mathrm{~K}$, the spectrum became a single narrow band [25]. This variation can be explained with the effect of magnetic exchange coupling [20,25]. The $J_{0}$ and $J_{1}$ values of tri$\operatorname{Cr}$ (III) acetate are: $J_{0}=10 \mathrm{~cm}^{-1}$ and $J_{1}=2.6 \mathrm{~cm}^{-1}$ [23]. The small $J_{1}$ value indicates that the $J_{1}$ coupling is unable to change the sequence of the spin energy levels predicted by the isotropic antiferromagnetic coupling. But, it is still able to remove the degeneracy of individual levels. For instance, the four-fold degenerate ground state of tri-chromium acetate is split into two spin doublets $\left(S^{\prime}=1 / 2\right)$ [20]. At the relatively high temperatures (e.g., 300 and $77 \mathrm{~K}$ ), the states of higher energy levels are populated and multiple transition paths become available, thereby producing a broadened band with multiple shoulders. At the low temperature (4.2 K), only two lower spin doublets resulting from the splitting of the ground state are accessed. The energy difference between these two doublets is too narrow to be resolved, resulting in a single band [20].

The second compound is a tri-Cr(III) hydroxo-bridged ammine complex $\left.\left(\left[\mathrm{Cr}_{3}\left(\mathrm{NH}_{3}\right)_{10}(\mathrm{OH})_{4}\right)\right] \mathrm{Br}_{5} \bullet 3 \mathrm{H}_{2} \mathrm{O}\right)$ [29]. In this molecule, three $\mathrm{Cr}(\mathrm{III})$ ions are bridged through four hydroxyl groups with an irregular triangle configuration like that in Scheme IIc. Magnetic properties of this compound have been studied by Andersen et al. [29]. The measured $J_{0}$ and $J_{1}$ values have suggested that the ground state and the lowest excited state of spin are a quartet $\left(S^{\prime}=\right.$ 
3/2) and a doublet $\left(S^{\prime}=1 / 2\right)$, respectively. The EPR spectra obtained at varied temperatures have also supported the above spin states. They show that with the decrease of temperature from $41 \mathrm{~K}$ to $3.0 \mathrm{~K}$, spin transitions vary from an isotropic doublet transition $(g \sim 1.98)$ to anisotropic quartet transitions. Two separate bands have been observed at the temperature of $3.0 \mathrm{~K}$.

The early work on these two compounds has indicated that they hold very different spin ground states, thereby yielding different EPR spectra at extremely low temperatures. The difference in the ground state is primarily attributed to their different exchange coupling which is dictated by the bridging configurations of tri-Cr(III) compounds. The EPR of trimer obtained in this work follows the pattern of the second compound but contradicts that of the first one. Therefore, we came to the conclusion that the trimer isolated by chromatography in this work has an irregular triangular configuration as shown in Scheme 2c.

\subsection{EXAFS}

Figure 3 shows the raw $k^{3}$-weighted EXAFS data and the corresponding Fourier Transform (FT) of the isolated monomer, dimer, and trimer. The FT represents a pseudoradial distribution function. Since no corrections were made on phase shifts, the FT peaks are shifted to a lower radial value $(\sim 0.2-0.5 \AA)$. The phase shifts are typically associated with the absorberscatter interactions. The theoretical curve fits are also depicted in the figure. Good fits were obtained for all the oligomers.

The structural parameters from the EXAFS fittings are listed in Table 2, including the parameters obtained for solid samples in this work and those for solution samples in early work [13]. For each oligomer, similar structural parameters were yielded for both forms of the samples. 
This confirmed that structures of the $\mathrm{Cr}$ (III) oligomers did not change throughout the preparation of solid samples from isolated oligomer solutions.

It is also noticed that for both $\mathrm{Cr}-\mathrm{O}$ and $\mathrm{Cr}-\mathrm{Cr}$ interactions, radial values from the solid samples in this work are slightly larger than those from the solution samples in the early work, consistently for all three oligomers (Table 2). This discrepancy may come from the difference in software for the data reduction and reference materials for the FEFF fitting. The R-space X-ray Absorption Package (RSXAP) [30] was used in the early work [13] and the crystal structures of grimaldite $(\alpha-\mathrm{CrOOH})$, guyanaite $(\beta-\mathrm{CrOOH})$ and a $\mathrm{Cr}(\mathrm{III})$ dimer compound [31] were used as reference compounds in the FEFF calculations. In this work, as mentioned in the experimental section, the EXAFSPAK suite was employed for the data treatments and the self-defined structures of monomer $\left(\left(\mathrm{H}_{2} \mathrm{O}\right)_{6} \mathrm{Cr}^{3+}\right)$ and dimer $\left(\left(\mathrm{H}_{2} \mathrm{O}\right)_{4} \mathrm{Cr}(\mathrm{OH})_{2} \mathrm{Cr}\left(\mathrm{H}_{2} \mathrm{O}\right)_{4}{ }^{4+}\right)$ for the FEFF calculations.
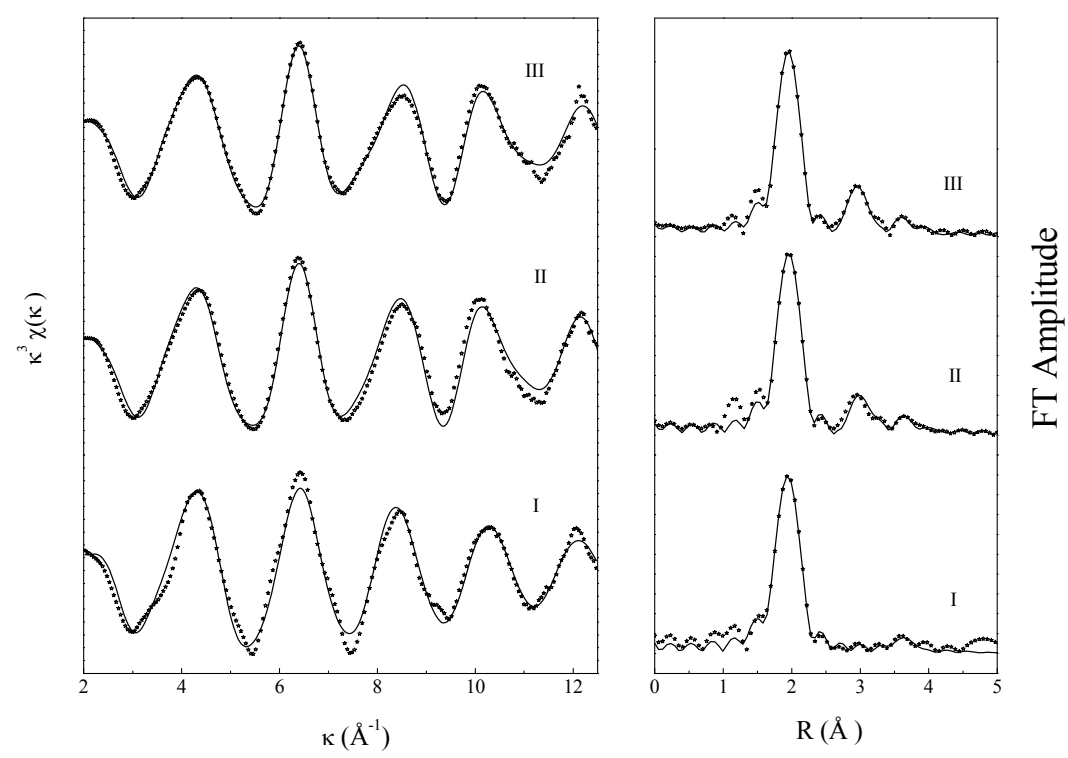

Figure 3 EXAFS results of Cr(III) oligomers. Monomer (I), dimer (II) and trimer (III). 


\subsubsection{Monomer EXAFS}

The EXAFS data of the monomer indicate that there are six oxygen atoms coordinating to the $\mathrm{Cr}$ (III) center at the distance of $1.98 \AA$ (c.f., Table 1). This is consistent with the result for the hydrated $\mathrm{Cr}$ (III) ion in aqueous solutions, which was obtained by EXAFS and LAXS (Large Angle X-ray Scattering) [32]. The result indicates that six oxygen atoms are located at $1.97 \AA$ around the $\mathrm{Cr}(\mathrm{III})$ ion (c.f., Scheme Ia). It also agrees with the assumption made in the discussion of monomer EPR, which is that six oxygen atoms at the apices of an octahedral structure form an octahedral crystal field to $\mathrm{Cr}(\mathrm{III})$.

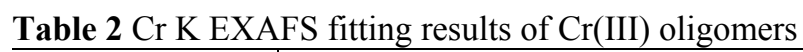

\begin{tabular}{|c|c|c|c|c|c|c|}
\hline Sample & Shell & $N^{\mathrm{a}}$ & $\begin{array}{l}R^{\mathrm{a}} \\
(\AA))\end{array}$ & $\begin{array}{c}\sigma^{2 a} \\
\left(\AA^{2}\right)\end{array}$ & $\begin{array}{l}\Delta E_{0} \\
(\mathrm{eV})\end{array}$ & $F$ \\
\hline \multirow[t]{2}{*}{ Monomer } & $\mathrm{Cr}-\mathrm{O}$ & 6.0 & 1.98 & 0.0049 & -6.2 & 0.19 \\
\hline & & $(5.9)^{b}$ & $(1.97)$ & $(0.0027)$ & & \\
\hline \multirow[t]{4}{*}{ Dimer } & $\mathrm{Cr}-\mathrm{O}$ & 6.0 & 1.99 & 0.0043 & -4.7 & 0.17 \\
\hline & & (5.9) & (1.94) & $(0.0045)$ & & \\
\hline & $\mathrm{Cr}-\mathrm{Cr}$ & 0.9 & 3.02 & 0.0038 & & \\
\hline & & $(0.8)$ & (2.99) & $(0.0031)$ & & \\
\hline \multirow[t]{4}{*}{ Trimer } & $\mathrm{Cr}-\mathrm{O}$ & 5.9 & 1.98 & 0.0047 & -5.2 & 0.14 \\
\hline & & $(5.8)$ & (1.94) & $(0.0037)$ & & \\
\hline & $\mathrm{Cr}-\mathrm{Cr}$ & 1.7 & 3.05 & 0.0055 & & \\
\hline & & (1.9) & (2.98) & $(0.0045)$ & & \\
\hline
\end{tabular}

a Those values were estimated by EXAFSPAK with the $95 \%$ confidence limits. Monomer: $\mathrm{Cr}-\mathrm{O}, N \pm 0.1, R \pm 0.002$ $\AA, \sigma^{2} \pm 0.0002$. Dimer: $\mathrm{Cr}-\mathrm{O}, N \pm 0.1, R \pm 0.002 \AA, \sigma^{2} \pm 0.0002$; $\mathrm{Cr}-\mathrm{Cr}, N \pm 0.1, R \pm 0.004 \AA, \sigma^{2} \pm 0.0009$. Trimer: $\mathrm{Cr}-\mathrm{O}, N \pm 0.1, R \pm 0.002 \AA, \sigma^{2} \pm 0.0002 ; \mathrm{Cr}-\mathrm{Cr}, N \pm 0.2, R \pm 0.003 \AA, \sigma^{2} \pm 0.0007$.

${ }^{\mathrm{b}}$ The values in parenthesis are the results of oligomer solution samples (ref. 13). The $\mathrm{pH}$ of those solutions is: 2.0 (monomer), 2.8 (dimer) and 2.8 (trimer).

\subsubsection{Dimer EXAFS}


The FT result of the dimer indicates the existence of two scattering paths around the central Cr atom (cf. Figure 3-II). In addition to a Cr-O scattering path $(N=6.0$ and $R=1.99 \AA)$, the presence of a Cr-Cr scattering path $(N=0.9$ and $R=3.02 \AA)$ confirms its di-Cr(III) configuration.

Two representative $\mathrm{Cr}-\mathrm{O}$ bond lengths and a $\mathrm{Cr}-\mathrm{Cr}$ distance of the dimer, which are obtained from the reported crystal structure [31], are depicted in Scheme Ib. It is clear that two Cr-O shells (1.94 and $2.00 \AA)$ are too close to be resolved from the EXAFS analysis. But, the average bond length $(1.98 \AA)$ excellently matches the $R_{\mathrm{Cr}-\mathrm{O}}$ value $(1.99 \AA)$ from the EXAFS (average bond length $=(8 \times 2.00+4 \times 1.94) / 12=1.98 \AA)$. Also, the Cr-Cr distance $(3.01 \AA)$ very well agrees with the $R_{\mathrm{Cr}-\mathrm{Cr}}(3.02 \AA)$. Those agreements suggest that the dime holds the structure as shown in Scheme Ib.

For the dimer structure, assuming that two bridging hydroxyl groups and two $\mathrm{Cr}$ (III) ions are located in the same plane, the O-Cr-O angle along the $\mathrm{Cr}-\mathrm{Cr}$ axis could be calculated to be $78^{\circ}$ from the $\mathrm{Cr}-\mathrm{O}$ and $\mathrm{Cr}-\mathrm{Cr}$ distances (1.94 $\AA$ and $3.01 \AA$, respectively). This angle is significantly smaller than the regular angle $\left(90^{\circ}\right)$ in a perfect octahedral symmetry, suggesting that the bridging stretches the dimer structure along the $\mathrm{Cr}-\mathrm{Cr}$ axis.

\subsubsection{Trimer EXAFS}

The FT magnitude of the trimer (Figure 3-III) indicates that the scattering intensity of the second shell $(\mathrm{Cr}-\mathrm{Cr})$ at $\sim 3.0 \AA$ becomes significantly higher for the trimer than that for the dimer. The fitting yielded $N_{\mathbf{C r}-\mathbf{C r}}=1.7 \pm 0.2$ at $R=3.05 \pm 0.1 \AA$. Even with the consideration of uncertainty of \pm 0.2 in $N_{\mathbf{C r}-\mathbf{C r}}$, the result denies a linear configuration (c.f., Scheme IIa) proposed for the trimer structure because the average $N_{\mathbf{C r}-\mathbf{C r}}$ in the linear configuration should be 1.33. 
Table 3 lists $\mathrm{Cr}-\mathrm{Cr}$ distances of three tri-Cr(III) crystalline compounds which have been referred to and/or discussed earlier. In the ligand-capped trimer compound [12], trimer holds an irregular triad configuration as shown in Scheme IIc. For comparison, the key crystal structural parameters of trimer are denoted in the scheme, including four representative $\mathrm{Cr}-\mathrm{O}$ bond lengths and three $\mathrm{Cr}-\mathrm{Cr}$ distances. It is clear that the EXAFS resolved coordination number $\left(N_{\mathrm{Cr}-\mathrm{O}}=5.9 \pm\right.$ $0.1)$ and radial value $\left(R_{\mathrm{Cr}-\mathrm{O}}=1.98 \pm 0.01 \AA\right)$ for the $\mathrm{Cr}-\mathrm{O}$ shell agree with the trimer structure in the compound. For the $\mathrm{Cr}-\mathrm{Cr}$ shell, the $N_{\mathrm{Cr}-\mathrm{Cr}}$ value $(1.7 \pm 0.2)$ supports a triangular configuration. But, the $R_{\mathrm{Cr}-\mathrm{Cr}}$ value $(3.05 \pm 0.1 \AA)$ appears interesting. It is much shorter than the average $\mathrm{Cr}-\mathrm{Cr}$ distance in the ligand-capped trimer compound (3.31 $\AA$, Table 3$)$. This may suggest that the structure of trimeric $\mathrm{Cr}(\mathrm{III})$ hydrolytic oligomer without the cap-ligand is more compacting.

Table $3 \mathrm{Cr}-\mathrm{Cr}$ distances of tri-Cr(III) crystalline compounds reported in literature

\begin{tabular}{|c|c|c|c|c|}
\hline Compound & Bridging & Distance $(\AA)$ & $\begin{array}{l}\text { Configuration } \\
\text { (Tri-Cr(III) ions) }\end{array}$ & Reference \\
\hline $\begin{array}{c}\operatorname{Tri}-\mathrm{Cr}(\mathrm{III}) \text { acetate } \\
\left(\left[\mathrm{Cr}_{3} \mathrm{O}\left(\mathrm{CH}_{3} \mathrm{COO}\right)_{6}\left(\mathrm{H}_{2} \mathrm{O}\right)_{3}\right] \mathrm{Cl} \cdot 6 \mathrm{H}_{2} \mathrm{O}\right)\end{array}$ & Carboxylate group $\& \mu_{3}-\mathrm{O}$ & 3.28 & Regular triad & {$[23,26,27]$} \\
\hline $\begin{array}{c}\text { Tri-Cr(III) amine complex } \\
\left.\left(\left[\mathrm{Cr}_{3}\left(\mathrm{NH}_{3}\right)_{10}(\mathrm{OH})_{4}\right)\right] \mathrm{Br}_{5} \cdot 3 \mathrm{H}_{2} \mathrm{O}\right)\end{array}$ & $\begin{array}{l}\text { Single-hydroxyl group } \\
\text { Double-hydroxyl groups } \\
(\text { Average })^{\mathrm{a}}\end{array}$ & $\begin{array}{c}3.00 \\
3.61 \\
(3.41)\end{array}$ & Irregular Triad & [29] \\
\hline $\begin{array}{l}\text { Ligand capped trimer } \\
\text { (ligand: } p \text {-sulfonatocalix[4,5]arenes) }\end{array}$ & $\begin{array}{c}\text { Single-hydroxyl group } \\
\text { Double-hydroxyl groups } \\
(\text { Average })^{\mathrm{a}}\end{array}$ & $\begin{array}{c}2.88 \\
3.51 / 3.58 \\
(3.31)\end{array}$ & Irregular Triad & [12] \\
\hline
\end{tabular}

${ }^{a}$ This average distance was calculated with the equation: Average distance $=(2 \mathrm{x}$ double-hydroxyl bridging distance $+1 \mathrm{x}$ single-hydroxyl bridging distance) / 3 . It is used for comparison to the EXAFS results.

The $\mathrm{Cr}$-Cr distances in the irregular triad compounds vary substantially from doublehydroxyl bridging to single-hydroxyl bridging (double/single: $2.88 / \sim 3.55 \AA$ and 3.00/3.61 $\AA$ for the ligand-capped trimer and the amine complex, respectively). For such a structure, the $\mathrm{Cr}-\mathrm{Cr}$ 
scattering could be readily resolved into two shells by the EXAFS analysis. However, from collected EXAFS data of our trimer sample, only one $\mathrm{Cr}$-Cr scattering path was obtained. This leads to the following: While the trimer EPR has demonstrated its irregular triad configuration, the geometric sizes of tri-(III) ions in the trimer may be quite different from those in the compounds of ligand capped trimer and the tri-Cr(III) amine complex. Most likely, the singlehydroxyl bridging in the trimeric $\mathrm{Cr}(\mathrm{III})$ hydrolytic oligomer brings two $\mathrm{Cr}(\mathrm{III})$ ions much more closely than does that in the compounds.

\section{Conclusions}

In this work, three $\mathrm{Cr}(\mathrm{III})$ oligomers in weakly acidic solutions, monomer, dimer, and trimer, were isolated by chromatography at $\mathrm{pH} \sim 2.5$. Their structures were studied by multiple spectroscopic techniques including UV-vis-IR, EPR and EXAFS. Integration of the data from these techniques has well demonstrated their structures.

It is the first time that the EPR spectra of $\mathrm{Cr}(\mathrm{III})$ hydrolytic oligomers were measured at extremely low temperatures $(1.4 \mathrm{~K})$. These spectra not only help elucidate their structures but also provide insight into their spin properties.

\section{Experimental}

\subsection{Chemicals}

All chemicals were of reagent grade or higher quality, and were used as received without further purifications. Deionized water from a Millipore system (18.3 M $\Omega)$ was used for the preparation of all aqueous solutions. 


\subsection{Isolation of oligomers}

A stock solution of $0.1 \mathrm{M} \mathrm{Cr}(\mathrm{III})$ was prepared by dissolving a known quantity of $\mathrm{Cr}\left(\mathrm{ClO}_{4}\right)_{3} \cdot 6 \mathrm{H}_{2} \mathrm{O}$ (Aldrich) in $0.1 \mathrm{M}$ perchloric acid. A working solution containing $\mathrm{Cr}(\mathrm{III})$ oligomers was obtained by mixing equal volumes of the $\mathrm{Cr}(\mathrm{III})$ stock solution and $0.1 \mathrm{M}$ sodium hydroxide with vigorous stirring. This solution was agitated mechanically for 2-3 days and then filtered with a $0.2 \mu \mathrm{m}$ nylon Acrodisc filter (Gelman). Sephadex SP C-25 cation exchange resin (Aldrich) was used for chromatographic separation of the oligomers. The resin was pretreated by immersion into $0.01 \mathrm{M} \mathrm{HClO}_{4}$ before it was loaded into a plastic column.

The procedures for the isolation of the Cr(III) oligomers have been described elsewhere [1]. In this work, the oligomers on the column were successively eluted by solutions of 0.01-0.04 $\mathrm{M} \mathrm{HClO}_{4}$ with increasing ionic strength from 1 to $4 \mathrm{M} \mathrm{NaClO}_{4}$. Fractions of the individual oligomers, including the monomer, dimer, and trimer, were well resolved by carefully controlling the elution rate and the timing of changing eluting agents. UV-visible absorption spectra of the separated fractions were measured on a Cary 5G spectrometer (Varian, Inc.). Absorbance features of these spectra were identical to those of the monomer, dimer and trimer, which were reported in the literature $[1,6-8]$, confirming that good separation was achieved. The solutions of the isolated $\mathrm{Cr}$ (III) oligomers were either immediately used for characterization tests or stored in a refrigerator for further use.

\subsection{EPR}

Electron Paramagnetic Resonance (EPR) spectroscopy was used to characterize the three isolated $\mathrm{Cr}$ (III) oligomers. For each oligomer solution, the spectra were measured at two 
different temperatures $(77$ and $1.4 \mathrm{~K})$. The chromium concentrations in these solutions were estimated to be around 1-5 $\mathrm{mM}$ from the absorption spectra of individual oligomers.

The EPR measurements at $\mathrm{T}=77 \mathrm{~K}$ were conducted at Washington State University on a Bruker 300e EPR spectrometer (Bruker Instruments, Inc.) with liquid nitrogen fluxing the sample chamber, while the experiments at $1.4 \mathrm{~K}$ were conducted on an EPR apparatus at Lawrence Berkeley National Laboratory. The parameters of the instrument were set as follows: center field $=2700 \mathrm{G}$, sweep width $=4000 / 4600 \mathrm{G}$, sweep number $=5$, microwave frequency $=$ $9.375 \mathrm{GHz}$, microwave power $=9.985 \mathrm{~mW}$, receiver gain $=4.0 \times 10^{4}$, and modulation frequency $=100 \mathrm{kHz}$. At the extremely low temperature $(1.4 \mathrm{~K})$, a separated EPR apparatus was used with liquid helium fluxing the sample chamber.

\subsection{EXAFS}

The structures of isolated $\mathrm{Cr}(\mathrm{III})$ oligomers were analyzed with Extended X-ray Absorption Fine Structure (EXAFS) spectroscopy. To use a transmission signal, a solid sample was prepared with the procedures described below. An oligomer was first precipitated out by adding a protonated pyridine buffer solution $(\mathrm{pH} \sim 5.5)$ to an isolated oligomer solution $[31,33]$. And then, the precipitate was recovered by ultrafiltration, washed twice with water and acetone, and dried briefly in a stream of air to remove acetone. All these operations were conducted as rapidly as possible at temperature below $0{ }^{\circ} \mathrm{C}$ to minimize the possibility of oligomer structurally changing. At the end, the EXAFS sample was obtained by mixing an appropriate amount of the dried precipitate with boron nitride and loading the mixture to an aluminum holder with a rectangular opening of $20 \mathrm{~mm}(\mathrm{~L}) \times 2 \mathrm{~mm}(\mathrm{~W})$ and a thickness of 0.5-1.0 $\mathrm{mm}$. 
The chromium K-edge EXAFS data of individual oligomers were collected at the Stanford Synchrotron Radiation Laboratory (SSRL) on wiggler beamline 4-1 under normal ring operating conditions $(3.0 \mathrm{GeV}, 50-100 \mathrm{~mA})$. Energy scans of the polychromatic X-ray beam were obtained using a $\mathrm{Si}(220)$ double-crystal monochromator. The vertical slit width was 0.5 $\mathrm{mm}$, which reduces the effects of beam instabilities and monochromator glitches while providing ample photon flux. The higher order harmonic content of the beam was reduced by detuning the crystals in the monochromator so that the incident flux was reduced to $50 \%$ of its maximum at the scan ending energy.

The EXAFS data reduction was performed by standard methods reviewed elsewhere using the suite of programs EXAFSPAK [34]. The spectra were energy-calibrated by simultaneously measuring the spectrum of the reference (chromium foil), i.e., assigning the first inflection point of the $\mathrm{K}$ absorption edge of chromium to be $5989 \mathrm{eV}$. The data reduction included pre-edge background subtraction followed by spline fitting and normalization to extract EXAFS data above the threshold energy, $E_{0}$. The curve fitting analyses were also conducted using EXAFSPAK to fit the raw $k^{3}$-weighted EXAFS data.

The data fit utilized the theoretical phases and amplitudes calculated by the program FEFF7 [35] with two self-defined model compounds, monomer $\left(\left(\mathrm{H}_{2} \mathrm{O}\right)_{6} \mathrm{Cr}^{3+}\right)$ and dimer $\left(\left(\mathrm{H}_{2} \mathrm{O}\right)_{4} \mathrm{Cr}(\mathrm{OH})_{2} \mathrm{Cr}\left(\mathrm{H}_{2} \mathrm{O}\right)_{4}^{4+}\right)$, in which a chromium atom is surrounded by six oxygens with the Cr-O distance of $1.99 \AA$, forming a regular octahedral structure. The monomer model was used to fit the monomer data and the dimer model to fit the data of both dimer and trimer. All the interactions included in the fits were derived from the single- or multiple-scattering (SS or MS) paths calculated from these compounds. The relevant paths included in the fits are SS Cr-O, SS Cr-Cr (only for dimer and trimer), MS cis-Cr-O-O (3-legged path) and MS trans-O-Cr-O (3/4- 
legged path). The amplitude reduction factor, $\mathrm{S}_{0}^{2}$, was held fixed at 0.9 for all of the fits. The shift in threshold energy, $\Delta E_{0}$, was allowed to vary as a global parameter in each of the fits.

\subsection{IR}

Infrared (IR) spectroscopy was used to characterize isolated $\mathrm{Cr}$ (III) oligomers. Using the same procedures as those used in the preparation of EXAFS solid samples, we obtained an oligomer precipitate and then mixed it with dry potassium bromide powder and ground in a mortar. Prior to measurements, the mixture was compressed in a die to form a pellet and the pellet then mounted on a sample holder. The IR spectra were recorded under dry nitrogen purging at $298 \pm 1 \mathrm{~K}$ on a Sirius $100 \mathrm{FT}-\mathrm{IR}$ spectrometer (Mattson Instruments, Inc.) with a 2 $\mathrm{cm}^{-1}$ resolution and 256 scans.

\section{Acknowledgements}

The experimental work conducted at Washington State University (WSU) was supported by US Department of Energy (DOE), Environmental Management Science Program (EMSP) under Contract No. FG0798ER-14930. The experimental work conducted at Lawrence Berkeley National Laboratory (LBNL) was supported by US DOE, EMSP under Contract No. DE-AC0205CH11231. Z. Zhang and S. B. Clark (WSU) and L. Rao (LBNL) acknowledge the support in the preparation of this manuscript by US DOE, the Heavy Element Chemistry Program under Contract No. DE-SC-0004102 at WSU and Contract No. DE-AC02-05CH11231 at LBNL, respectively. The authors thank Dr. Wayne Lukens at LBNL for help in EPR experiments at extremely low temperatures. Stanford Synchrotron Radiation Laboratory (SSRL) is a user facility supported by US DOE, Office of Science, Office of Basic Energy Sciences. 


\section{References}

[1] H. Stünzi, W. Marty, Inorg. Chem. 22 (1983) 2145.

[2] L. Spiccia, W. Marty, Inorg. Chem. 25 (1986) 266-271.

[3] M. Ardon, R. A. Plane, J. Am. Chem. Soc. 81 (1959) 3197.

[4] J. A. Laswick, R. A. Plane, J. Am. Chem. Soc. 81 (1959) 3564.

[5] R. W. Kolaczkowski, R. A. Plane, Inorg. Chem. 3 (1964) 322-324.

[6] M. Thompson, R. E. Connick, Inorg. Chem. 20 (1981) 2279.

[7] J. E. Finholt, M. E. Thompson, R. E. Connick, Inorg. Chem. 20 (1981) 4151.

[8] H. Stünzi, F. P. Rotzinger, W. Marty, Inorg. Chem. 23 (1984) 2160.

[9] F. P. Rotzinger, H. Stünzi, W. Marty, Inorg. Chem. 25 (1986) 489.

[10] H. Stünzi, L. Spiccia, F. P. Rotzinger, W. Marty, Inorg. Chem. 28 (1989) 66.

[11] N. Bjerrum, Ph.D. Thesis, Copenhagen, 1908.

[12] A. Drljaca, M. J. Hardie, C. L. Raston, L. Spiccia, Chem. Eur. J. 5 (1999) 2295.

[13] L. Rao, Z. Zhang, J. I. Friese, B. Ritherdon, S. B. Clark, N. J. Hess, D. Rai, Journal of the Chemical Society, Dalton Transactions, (2) (2002) 267.

[14] J. I. Friese, B. Ritherdon, S. B. Clark, Z. Zhang, L. Rao, D. Rai, Analytical Chemistry, 74(13) (2002) 2977.

[15] L. L. Lohr, Coord. Chem. Rev. 8 (1972) 341

[16] R. S. Drago, In Physical Methods in Chemistry; Saunders: Philadelphia, 1977; Chapters 9, 10 and 13.

[17] C.K. Jorgensen, Acta Chem. Scand. 8 (1957) 1686.

[18] A. Abraham, B. Bleaney, In Electron Paramagnetic Resonance of Transition Ions; Dover Publications, Inc.: New York, 1986; pp 431-432.

[19] K. Kambe, J. Phys. Soc. Japan 5 (1950) 48.

[20] V. A. Gaponenko, M. V. Eremin, Yu. V. Yablokov, Sov. Phys. Solid State 15(5) (1973) 909.

[21] A. Das, J. C. Jeffery, J. P. Maher, J. A. McCleverty, E. Schatz, M. D. Ward, G. Wollermann, Inorg. Chem. 32 (10) (1993) 2145.

[22] F. Birkelbach, M. Winter, U. Florke, H. Haupt, C. Butzlaff, M. Lengen, E. Bill, A. X. Trautwein, K. Wieghardt, P. Chaudhuri, Inorg. Chem. 33 (1994) 3990.

[23] B. N. Figgis, G. B. Robertson, Nature 205 (1965) 694.

[24] L. Dubicki, P. Day, Inorg. Chem. 11 (1972) 1868.

[25] Yu. V. Yablokov, V. A. Gaponenko, V. V. Zelentsov, Sov. Phys. Solid State 13(5) (1971) 1081.

[26] S.C. Chang, G. A. Jeffrey, Acta Crystallogr., Sect. B 26 (1970) 673.

[27] C. E. Anson, J. P. Bourke, R. D. Cannon, U. A. Jayasooriya, M. Molinier, A. K. Powell, Inorg. Chem. 36 (1997) 1265.

[28] Yu. V. Yablokov, V. A. Gaponenko, A. V. Ablov, T. N. Zhikhareva, Sov. Phys. Solid State 15(2) (1973) 251.

[29] P. Andersen, T. Damhus, E. Pedersen, A. Petersen, Acta Chemica Scandinavica A 38 (1984) 359

[30] G. G. Li, F. Bridges, C. W. Booth, Phys. Rev. B 52 (1995) 6332

[31] L. Spiccia, H. Stoeckli-Evans, W. Marty, R. Giovanoli, Inorg. Chem. 26 (1987) 474. 
[32] P. Lindqvist-Reis, A. Munoz-Paez, S. Diaz-Moreno, S. Pattanaik, I. Persson, M. Sandstrom, Inorg. Chem. 37 (1998) 6675.

[33] L. Spiccia, W. Marty, R. Giovanoli, Inorg. Chem. 27 (1988) 2660.

[34] D. E. Koningsberger, R. Prins, X-ray Absorption: Principles, Applications, Techniques for EXAFS, SEXAFS, and XANES; Wiley-Interscience: New York, 1988.

[35] S. I. Zabinsky, J. J. Rehr, A. Ankudinov, R. C. Albers, M. J. Eller, Phys. Rev. B 52 (1995) 2995. 
Graphical Abstract
structural analysis of
Cr(III) oligomers

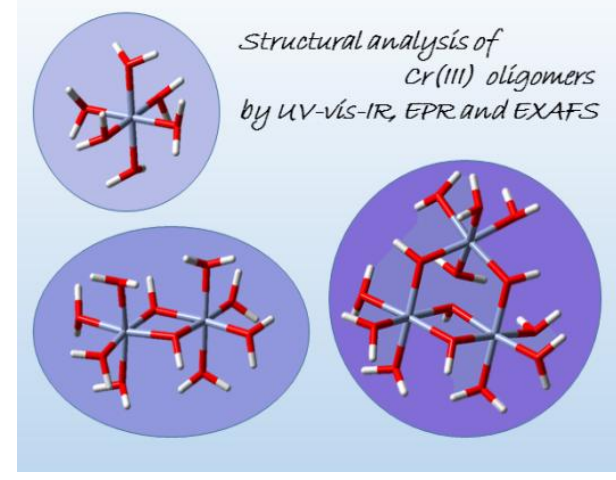

